Draft Version November 13, 2018

Preprint typeset using $\mathrm{IAT}_{\mathrm{E}} \mathrm{X}$ style emulateapj v. 5/2/11

\title{
BLACK HOLE TRIPLE DYNAMICS: BREAKDOWN OF THE ORBIT AVERAGE APPROXIMATION AND IMPLICATIONS FOR GRAVITATIONAL WAVE DETECTIONS
}

\author{
Fabio Antonini ${ }^{a}$, Norman Murray $^{a} \&$ Seppo Mikkola ${ }^{b}$ \\ ${ }^{a}$ Canadian Institute for Theoretical Astrophysics, University of Toronto, 60 George St., Toronto, Ontario \\ M5S 3H8, CANADA \\ ${ }^{b}$ Tuorla Observatory, University of Turku, Väisäläntie 20, Pinkkiö Fi-21500, Finland \\ E-MAIL: antonini@cita.utoronto.ca \\ Draft version November 13, 2018
}

\begin{abstract}
Coalescing black hole (BH) binaries forming via dynamical interactions in the dense core of globular clusters (GCs) are expected to be one the brightest and most numerous sources of gravitational wave $(\mathrm{GW})$ radiation, detectable by the upcoming generation of ground based laser interferometers. Favorable conditions for merger are initiated by the Kozai resonance in which the gravitational interaction with a third distant object, typically another $\mathrm{BH}$, induces quasi-periodic variations of the inner BH binary eccentricity. In this paper we perform high precision 3-body simulations of the long term evolution of hierarchical $\mathrm{BH}$ triples and investigate the conditions that lead to the merging of the $\mathrm{BH}$ binary and the way it might become an observable source of GW radiation. We find that the secular orbit average treatment, adopted in previous works, does not reliably describe the dynamics of these systems if the binary is orbited by the outer $\mathrm{BH}$ on a highly inclined orbit with a periapsis distance less than $\sim 10$ times the inner binary semi-major axis. During the high eccentricity phase of a Kozai cycle the torque due to the outer BH can drive the binary to extremely large eccentricities in a fraction of the binary's orbital period. This occurs before relativistic terms become important to the evolution and allows the binary GW signal to reach large GW frequencies ( $\gtrsim 10 \mathrm{~Hz})$ at high eccentricities. We show that $30-50 \%$ of coalescing BH binaries driven by the Kozai mechanism in GCs will have eccentricities larger than 0.1 , with $10 \%$ of them being extremely eccentric, $(1-e)<10^{-5}$, when they first chirp in the frequency band of ground based laser interferometers. This implies that a large fraction of such GW sources could be missed if conventional quasi-circular templates are used for analysis of GW detectors data. The efficient detection of all coalescing BH binaries in GCs will therefore require template banks of eccentric inspiral waveforms for matched-filtering and dedicated search strategies. We conclude by noting that our results have potential application to a variety of astrophysical systems, including type Ia supernovae, X-ray binaries, optical transients and their merger products.
\end{abstract}

Subject headings: Gravitational waves - binaries - globular clusters - stars: kinematics and dynamics

\section{INTRODUCTION}

Coalescence of compact binaries is accompanied by the emission of gravitational waves (GWs) at frequencies that are potentially detectable by the next generation of ground-based laser interferometers. Indeed, the first GW signature from a compact binary coalescence could be detected as early as 2015 when the advanced version of the GW observatories advancedLIGO (aLIGO) 1 and advanced-VIRGO2 2 will become operative (Acernese et al. 2008; Harry et al. 2010). The aLIGO detectors will be able to see inspiraling neutron stars (NSs) up to a horizon distance of about $400 \mathrm{Mpc}$, NS-black hole (BH) binaries will be visible to about 900 $\mathrm{Mpc}$, and coalescing $\mathrm{BH}$ binary systems will be visible to cosmological distances, up to a few Gpc (Abadie et al. 2010). This would provide a unique way to test General Relativity in the dynamical strong-field regime (so far unverified by direct observations), and shed some light on astrophysically interesting properties of the de-

\footnotetext{
1 http://www.ligo.caltech.edu

2 https://wwwcascina.virgo.infn.it/advirgo/
}

tected sources like their masses, spins and birth rate (e.g., Abramovic et al. 1992).

At present, astrophysical predictions for compact binary coalescence rates remain highly uncertain (Abadie et al. 2010). Rate estimates mostly relay on population synthesis models which are based on a number of assumptions and poorly constrained model parameters (e.g., Naravan et al. 1991; Portegies Zwart \& Yungelson 1998; Belczynski et al. 2002; Kalogera et al. 2007). These models often predict that detections of GW sources from the galactic field will be dominated by NS binary inspirals while BH binaries will only be a small contributor to the total rate (about 1 in 10 detections; O'Shaughnessy et al. 2007). This conclusion is based on results of stellar evolution calculations which indicate that formation of close $\mathrm{BH}$ pairs through evolution of massive stars in isolated binary systems is a rare phenomenon (Belczynski et al. 2007).

Although $\mathrm{BH}$ binaries might be rare in the Galactic field, it is possible that they efficiently form via dynamical interactions in dense stellar environments (Sigurdsson \& Phinney 1993) like for example 
galactic nuclei (Miller \& Lauburg 2009) and globular clusters (GCs) (O'Leary et al. 2006; Downing et al. 2010, 2011; Baneriee et al. 2010). In these dense stellar systems, otherwise rare dynamical processes can take place and affect the dynamical evolution of the $\mathrm{BH}$ population. Due to dynamical friction BHs tend to segregate (Spitzer 1987) toward the core of the cluster, or galaxy, where dynamical interactions with stars and other BHs will lead to the formation, hardening and (in some cases) ejection of $\mathrm{BH}$ binaries (Heggie 1975) before they can efficiently inspiral and coalesce in response to gravitational radiation. The merger rate for these binaries, although uncertain, could be as large as a few thousand events a year for aLIGO and be comparable or even greater than the event rate for NS binary mergers (e.g., Portegies Zwart \& McMillan 2000; Sadowski et al. 2008; Downing et al. 2011).

Since stellar $\mathrm{BH}$ binaries are not directly detected, detailed modeling of their formation processes and dynamical evolution is crucial for making predictions about their characteristic GW-form and in turn for enabling efficient detection with future GW observatories. It was first noted by Miller \& Hamilton (2002a) that binarybinary encounters play a decisive role in initiating favorable conditions for BH binary coalescence in GCs. A large fraction, roughly $20-50 \%$, of these encounters leave behind a stable hierarchical $(\mathrm{BH})$ triple system (e.g., Sigurdsson \& Hernquist 1993; Kulkarni et al. 1993).

If the orbital plane of the inner binary is strongly tilted with respect to the orbital plane of the outer object the secular Kozai mechanism can lead the inner binary orbit to very high eccentricities (Kozai 1962; Lidov 1962). In turn, given that the timescale associated with GW radiation is a strong function of eccentricity (Peters 1964), a combination of Kozai cycles and GW energy loss can enormously accelerate the merger of a compact binary (e.g., Blaes et al. 2002). A quite convincing demonstration of the importance of the Kozai mechanism in determining the conditions for $\mathrm{BH}$ binary mergers in stellar clusters was recently provided by Aarseth (2012) who made use of a hybrid $N$-body code incorporating the algorithmic regularization method of Hellström \& Mikkola (2010) and post-Newtonian forces to evolve GC models containing BHs and NSs. Aarseth finds that the onset of conditions for GW radiation is usually initiated by the Kozai mechanism.

A number of papers have considered the Kozai resonance as a mechanism for accelerating compact object mergers in triple systems (e.g., Blaes et al. 2002; Miller \& Hamilton 2002a; Wen 2003; Thompson 2011; Prodan et al. 2013a). These authors dealt with the problem by averaging over orbital phases, i.e., averaging the equations of motion over the short timescales associated with the unperturbed Keplerian motions of both inner and outer orbit. The result is a set of first-order differential equations that describe the long-term (secular) evolution of the remaining orbital elements due to the perturbing forces (Merritt 2013).

In this paper we perform direct integrations of the post-Newtonian equations of motion describing triple systems. We do this by using the AR-CHAIN code which is able to trace the motion of tight binaries with arbitrary mass ratio for long periods of time with extremely high precision (Mikkola \& Merritt 2006, 2008). The code combines the use of the chain regularization method of Mikkola \& Aarseth (1993) and the time transformed leapfrog scheme to avoid singularities. This allows for an essentially exact treatment of the dynamics avoiding the approximations that are made when using the orbit average treatment, at expense, of course, of computational efficiency. The employment of an $N$-body integrator is motivated by a recent study of Antonini \& Perets (2012) who have shown, in the context of $\mathrm{BH}$ binary mergers in galactic nuclei, that the orbit averaged approximation breaks down for highly inclined but stable configurations if the binary comes closer to the outer perturber than a certain distance (see $\S 4.1$ of Antonini \& Perets 2012, or Katz \& Dong 2013 and Seto 2013 for an application of this in the context of white dwarf-white dwarf and NS-NS collisions respectively).

In this work we investigate the dynamics of coalescing $\mathrm{BH}$ binaries driven by the Kozai mechanism and focus on their eccentricity distribution when the GW signal reaches frequencies that will be detectable by the next generation of ground-based laser interferometers. Similar analysis were previously conducted by Miller \& Hamilton (2002a), Wen (2003) and O'Leary et al. (2006). These previous papers employed the standard orbit average approximation, which we demonstrate to be inaccurate in describing the dynamics of such systems even when including octupole order terms in the evolution. Our $N$-body experiments reveal that inspiraling $\mathrm{BH}$ binaries often reach the gravitational-radiation-dominated regime while on orbits that are still very eccentric; eccentricities can be large enough that detecting the GW signal from these sources will require the use of eccentric templates for data analysis and dedicated search strategies (East et al. 2013).

In $\S 2$ we review the concepts behind the classic Kozai secular theory for the evolution of hierarchical triples noting the simplifying approximations that are made in this treatment. In $\S 3$ we show that the standard orbit average approximation breaks down if the inner binary is orbited by an outer perturber with a moderate periapsis, and determine an approximate condition for this to occur. In $\S 4$ we make use of direct numerical threebody integrations to study the dynamics of hierarchical $\mathrm{BH}$ triples in GCs and focus on the evolution of the inner $\mathrm{BH}$ binary into a short period orbit and the way in which it may become an observable GW source for the advanced GW detectors. We discuss and summarize our results in $\S 5$.

\section{ORBIT AVERAGED EQUATIONS}

We consider stellar $\mathrm{BH}$ binaries with components of masses $m_{0}$ and $m_{1}$ orbiting a third body of mass $m_{2}$. We denote the eccentricities of the inner and outer orbits, respectively, as $e_{1}$ and $e_{2}$, and semi-major axes $a_{1}$ and $a_{2}$. We define $\omega_{1}$ as the argument of periapsis of the inner binary relative to the line of the descending node, $\omega_{2}$ as the argument of periapsis of the outer orbit, and $I$ as the mutual orbital inclination of the inner orbit with respect to the outer orbit.

If the distance of the outer $\mathrm{BH}$ is much larger than the semi-major axis of the inner binary the dynamics of the entire system can be described as the interaction between an inner binary of point masses $m_{0}$ and $m_{1}$ and an external binary of masses $M_{b}=m_{0}+m_{1}$ and $m_{2}$. We 
define the angular momenta $L_{1}$ and $L_{2}$ of the inner and outer binary and total angular momentum $\mathbf{H}=\mathbf{L}_{1}+\mathbf{L}_{2}$ :

$$
L_{1}=m_{0} m_{1}\left[\frac{G a_{1}\left(1-e_{1}^{2}\right)}{M_{b}}\right]^{1 / 2},
$$

and

$$
L_{2}=M_{b} m_{2}\left[\frac{G a_{2}\left(1-e_{2}^{2}\right)}{M_{b}+m_{2}}\right]^{1 / 2},
$$

with $G$ the gravitational constant. We define the dimensionless angular momenta $\ell_{1}=L_{1} / L_{1, c}$ and $\ell_{2}=L_{2} / L_{2, c}$ with $L_{i, c}=L_{i} / \sqrt{1-e_{i}^{2}}$ the angular momentum of a circular orbit with the same $a_{i}$.

When changes in the orbital properties of a three-body system occur on a timescale longer than both the inner binary and the outer tertiary orbital periods, it is convenient to average the motion over both these periods. The resulting double average Hamiltonian of the system is $\mathcal{H}=k W$ with $k=3 G m_{0} m_{1} m_{2} a_{1}^{2} / 8 M_{1} a_{2}\left(1-e_{2}^{2}\right)^{1 / 2}$ and (Miller \& Hamilton 2002a)

$$
\begin{aligned}
W\left(\omega_{1}, e_{1}\right)= & -2\left(1-e_{1}^{2}\right)+\left(1-e_{1}^{2}\right) \cos ^{2} I \\
& +5 e_{1} \sin 2 \omega_{1}\left(\cos ^{2} I-1\right)+\frac{\chi}{\sqrt{1-e_{1}^{2}}},
\end{aligned}
$$

with

$$
\chi=\frac{8 M_{b}}{m_{2}}\left[\frac{a_{2}\left(1-e_{2}^{2}\right)}{a_{1}}\right]^{3} \frac{G M_{b}}{a_{1} c^{2}}
$$

and $c$ the speed of light.

The quadrupole-level secular perturbation equations can be easily derived from the conserved Hamiltonian (3). The resulting evolution equation of the inner binary orbital elements, including Schwarzschild precession (SP) and quadrupole gravitational wave radiation terms, are (Ford et al. 2000; Peters 1964):

$$
\begin{gathered}
\frac{d e_{1}}{d t}=\frac{30 K}{L_{1, c}} e_{1} \sqrt{1-e_{1}^{2}}\left(1-\cos ^{2} I\right) \sin 2 \omega_{1} \\
-\frac{304 G^{3} m_{0} m_{1} M_{b} e_{1}}{15 c^{5} a_{1}^{4}\left(1-e_{1}^{2}\right)^{5 / 2}}\left(1+\frac{121}{304} e_{1}^{2}\right) \\
\frac{d \omega_{1}}{d t}=\frac{6 K}{L_{1, c}}\left(\frac { 1 } { \sqrt { 1 - e _ { 1 } ^ { 2 } } } \left[4 \cos ^{2} I+\right.\right. \\
\left.\left(5 \cos 2 \omega_{1}-1\right)\left(1-e_{1}^{2}-\cos ^{2} I\right)\right] \\
\left.+\frac{L_{1, c} \cos I}{L_{2}}\left[2+e_{1}^{2}\left(3-5 \cos 2 \omega_{1}\right)\right]+\frac{\chi}{1-e_{1}^{2}}\right) \\
\frac{d a_{1}}{d t}=\frac{-64 G^{3} m_{0} m_{1} M_{b}}{5 c^{5} a_{1}^{3}\left(1-e_{1}^{2}\right)^{7 / 2}}\left(1+\frac{73}{24} e_{1}^{2}+\frac{37}{96} e_{1}^{4}\right) \\
\frac{d H}{d t}=-\frac{32 G^{3} m_{0}^{2} m_{1}^{2}}{5 c^{5} a_{1}^{3}\left(1-e_{1}^{2}\right)^{2}}\left[\frac{G M_{b}}{a_{1}}\right]^{1 / 2} \\
\left(1+\frac{7}{8} e_{1}^{2}\right) \frac{L_{1}+L_{2} \cos I}{H}
\end{gathered}
$$

where

$$
K=\frac{G m_{0} m_{1} m_{2}}{16 M_{b} a_{2}\left(1-e_{2}^{2}\right)^{3 / 2}}\left(\frac{a_{1}}{a_{2}}\right)^{2}
$$

and

$$
\cos I=\frac{H^{2}-L_{1}^{2}-L_{2}^{2}}{2 L_{1} L_{2}} .
$$

Equations (5)-(8) can be considered as describing the interaction between two weighted wires instead of point masses in orbits. Generally, these equations are applied to the dynamics of triple systems that satisfy the stability criterion (e.g., Blaes et al. 2002; Wen 2003; Fabrycky \& Tremaine 2007; Hamers et al. 2013):

$$
\begin{aligned}
\frac{a_{2}}{a_{1}}> & \frac{3.3}{1-e_{2}}\left[\frac{2}{3}\left(1+\frac{m_{2}}{M_{b}}\right) \frac{1+e_{2}}{\left(1-e_{2}\right)^{1 / 2}}\right]^{2 / 5} \\
& \times(1-0.3 I / \pi),
\end{aligned}
$$

which was derived in Mardling \& Aarseth (2001) by means of Newtonian direct $N$-body simulations. Systems that satisfy this criterion are stable hierarchical systems, meaning that the semi-major axis of the inner binary is constant on a secular timescale, in contrast to unstable systems that experience chaotic energy exchange which inevitably leads to the escape of one body over a short timescale.

The binary starts from initial eccentricity $e_{1}(0)$, semimajor axis $a_{1}(0)$, argument of periapsis $\omega_{1}(0)$ and mutual inclination $I(0)$ and evolves through interaction with the third body to a maximum eccentricity $e_{\max }$ and critical $\omega_{\text {crit }}$ and $I_{\text {crit }}$. For initially small inclinations, $60 \cos ^{2} \mathrm{I}(0) \gg \chi^{2}$, relativistic terms can be ignored and one finds $e_{\max }=\left[1-(5 / 3) \cos ^{2} \mathrm{I}(0)\right]^{1 / 2}$ Innanen et al. 1997). High initial inclinations will instead result in large values of $e_{\max }$ for which relativistic terms become relevant, for instance by limiting the maximum eccentricity attainable by the inner binary orbit. The influence of relativistic processes on the dynamical evolution of the $\mathrm{BH}$ triple are briefly discussed in what follows.

\subsection{The role of relativistic effects}

If the inner binary precesses "quickly", i.e., in a timescale short compared with the time for changes in $\mathbf{L}_{1}$, the resulting, averaged equations of motion will no longer contain $\omega_{1}$, and so they will conserve the momentum conjugate to $\omega_{1}$, which is $L_{1}$. This simple argument indicates that any mechanism inducing rapid apsidal precession, e.g., SP for an eccentric orbit, will suppress the Kozai resonance. However, if the relativistic precession timescales are comparable or lower than the secular Newtonian timescales, then around some critical value of $a_{2}$ it is possible that the maximum achievable eccentricity is increased with respect to the Newtonian case (Ford et al. 2000; Naoz et al. 2013).

For highly eccentric orbits, the SP (whose associated rate diverges as $\left(1-e_{1}\right)^{-1}$ ) sets a lower bound, $\ell_{\mathrm{SP}}$, to the angular momentum attainable by the inner binary orbit. The minimum angular momentum allowed can then be obtained from the quadrupole-order equations of motion (5)-(8) by setting $d e_{1} / d t=0$ and from conservation of $W\left(\omega_{1}, e_{1}\right)$ (e.g., Miller \& Hamilton 2002a). 
If we ignore energy loss due to GW emission, in the restricted three body problem (i.e., $m_{0} \gg m_{1} \gg m_{2}$ ), one finds (Miller \& Hamilton 2002a; Wen 2003):

$$
\ell_{\mathrm{SP}} \approx \sqrt{1-\left(\frac{\chi}{9}\right)^{2}}
$$

where $e_{1} \approx 1$, high initial inclination $(I(0) \approx \pi / 2)$, and weak precession $(\chi \ll 3)$ have been assumed.

The importance of SP to the evolution of the inner binary orbit depends on the role of GW emission which might dominate the evolution before $\ell_{\mathrm{SP}}$ is reached. From Equation (5), by setting $d e / d t=0$ and neglecting angular terms, we find that the critical angular momentum below which GW energy loss dominates the evolution is approximately:

$$
\ell_{\mathrm{GW}} \approx\left(\frac{G^{3} m_{0} m_{1} M_{b}}{c^{5} a_{1}^{4}} \frac{L_{1, c}}{K}\right)^{1 / 6}
$$

Transition between Kozai dynamics and GW driven inspiral occurs at

$$
\ell_{1}=\ell_{\mathrm{GW}}
$$

For $\ell_{G W}<\ell_{1}$, the inner binary "decouples" from the third body, and inspirals approximately as an isolated system. If $\ell_{\mathrm{GW}}>\ell_{\mathrm{SP}}, \mathrm{GW}$ radiation reaction will dominate the evolution within one Kozai cycle and the SP will be unimportant. In the (quadrupole-level) orbit average approximation this is a necessary condition for the binary to maintain a finite eccentricity as its GW frequency evolves through the aLIGO band.

Given the rapid orbital circularization due to GW emission we would expect $e_{1}$ to be typically small as the binary GW frequency passes through the high frequency band of ground based interferometers. On the other hand, it is possible that the binary GW frequency crosses the lower end of the aLIGO frequency band, $f_{\mathrm{GW}}^{L}=$ $10 \mathrm{~Hz}$, before $\mathrm{GW}$ radiation dominates the evolution, which will allow the eccentricity to be large $\left(\sim e_{\max }\right)$ at such high frequencies 3 . Such situation occurs only if $\ell\left(f_{\mathrm{GW}}^{L}\right)>\ell_{\mathrm{GW}}$, with $\ell\left(f_{\mathrm{GW}}^{L}\right)$ the binary angular momentum corresponding to the detector characteristic frequency and initial binary semi-major axis $a_{1}(0)$.

Eccentric binaries emit a GW signal with a broad spectrum of frequencies; the peak gravitational wave frequency corresponding to the harmonic which leads to the maximal emission of GW radiation can be approximated as (Wen 2003)

$$
f_{\mathrm{GW}}=\frac{\sqrt{G M_{b}}}{\pi} \frac{\left(1+e_{1}\right)^{1.1954}}{\left[a_{1}\left(1-e_{1}^{2}\right)\right]^{1.5}} .
$$

Under the quadrupole approximation the condition for the system to be able to pass through the detector frequency band before GW radiation dominates is

$$
\begin{aligned}
\frac{a_{2} \ell_{2}}{a_{1}} & <\left(\frac{2}{5} \frac{c^{5}}{\left(\pi f_{\mathrm{GW}}^{L}\right)^{2}} \frac{m_{2} M_{b}}{m_{0} m_{1}}\right)^{1 / 3} \frac{a_{1}^{-1 / 6}}{\left(G M_{b}\right)^{1 / 2}} \\
& =3.3\left(\frac{1}{2} \frac{m_{2} M_{b}}{m_{0} m_{1}}\right)^{1 / 3}\left(\frac{f_{\mathrm{GW}}^{L}}{10 \mathrm{~Hz}}\right)^{-2 / 3}
\end{aligned}
$$

3 http://www.ligo.caltech.edu/advLIGO/scripts/summary.shtml

$$
\times\left(\frac{M_{b}}{20 M_{\odot}}\right)^{-1 / 2}\left(\frac{a_{1}}{1 \mathrm{AU}}\right)^{-1 / 6} .
$$

By additionally requiring the triple system to be stable, Equations (11) and (16) imply that the range of initial conditions that would allow a $\mathrm{BH}$ binary to enter the aLIGO frequency band while on a high eccentric orbit is quite small. For instance, for the compact-object masses considered here, setting $a_{1}=0.3 \mathrm{AU}$ and $e_{2} \sim 0$, highly eccentric GW sources can only be produced for $2.8 \lesssim a_{2} / a_{1} \lesssim 4$. However, as we demonstrate in the next section, such a result is an artifact of the doubleaverage approximation which is shown to break down for $a_{2}\left(1-e_{2}\right) / a_{1} \lesssim 10$, i.e., well above the stability boundary implied by Equation (11). In fact, we find that in this region of parameter space, the system evolves through a complex dynamical evolution which can lead to arbitrarily large eccentricities before the fast orbital circularization due to GW loss begins. Our calculations predict that at least a few percent of $\mathrm{BH}$ binaries will have extremely high eccentricities while entering the aLIGO band.

\section{DIRECT NUMERICAL INTEGRATIONS}

\subsection{Numerical method}

We tested the applicability of the orbit-averaged approach for the study of GW sources by comparing the results of the orbit-averaged equations of motion (in both their quadrupole and octupole level form 4 , with those from numerical three-body integrations having the same initial conditions (and arbitrary orbital phases). The triple dynamics was followed until the inner binary $\mathrm{GW}$ signal reached the lower end of the aLIGO frequency band, which we identify here with the moment at which the binary peak GW frequency reaches the $10 \mathrm{~Hz}$ frequency.

We used the high accuracy $N$-body integrator ARCHAIN (Mikkola \& Merritt 2008), which includes postNewtonian (PN) non-dissipative $1 \mathrm{PN}, 2 \mathrm{PN}$ and dissipative 2.5PN corrections to all pair-forces. The code employs an algorithmically regularized chain structure and the time-transformed leapfrog scheme to accurately trace the motion of tight binaries with arbitrarily large mass ratios. This permits an essentially exact (at the PN level) treatment of the interplay between Newtonian and relativistic perturbations to the motion, avoiding the approximations that are made when using the orbit average equations of motion. We refer the reader to Mikkola \& Merritt (2006) and Mikkola \& Merritt (2008) for a more detailed description of AR-CHAIN.

Since following the inspiral of the binary all the way down to the aLIGO band is not practical with ARCHAIN, we stopped integrating the triple once the binary orbital separation had shrunk (because of GW energy loss) to about $10^{-2} \times a_{1}(0) 5$ At this point the

4 We integrated the equations of motion of Blaes et al. (2002) by using a $7 / 8$ order Runge-Kutta algorithm with a variable timestep (Fehlberg 1968) in order to keep the relative error per step in energy, in the absence of GW energy loss, less than $10^{-8}$. When GW radiation was included, we checked the integration accuracy through the quantity $E+E_{\mathrm{GW}}$ with $E$ the energy per unit mass and $E_{\mathrm{GW}}$ the work done by GW radiation along the trajectory. The accuracy in this case was of the same order of that found in integrations without dissipative terms.

5 The semi-major axis and eccentricity were calculated from 
binary dynamics is dominated by GW radiation and the influence of the third body can be safely neglected, so we continued evolving the binary eccentricity and semimajor axis by using the leading order orbit average analytical formulas of Peters (1964). This allowed for a more efficient computation of the dynamical evolution of the system.

\subsection{Breakdown of the orbit average approximation}

We begin by studying the eccentricity of the inner binary at the time its GW signal first enters the aLIGO frequency band, i.e. $f_{\mathrm{GW}}=10 \mathrm{~Hz}$, for a set of illustrative systems that are close to the stability criterion given by Equation (11) and for which the initial mutual inclination is large. It is from these marginally hierarchical configurations that we expect the larger number of eccentric sources for aLIGO, as well as the larger discrepancy between the results of $N$-body integrations and the predictions of the orbit average equations of motion (Antonini \& Perets 2012).

Two sets of initial conditions are explored in Figure 1 . In the upper panel we set $m_{0}=m_{1}=m_{2}=5 M_{\odot}$. In the secular theory the octupole order terms go to zero for $m_{0}=m_{1}$, and we are therefore left with the contribution from the quadrupole order terms only, i.e., Equations (5)(8).

Systems that are unstable according to Equation (11) (hatched region in Figure 11) experience the ejection of one component before the inner binary can complete one Kozai cycle and possibly merge. An example of such systems is the first point on the left in the upper panel of Figure 1, which is the same system shown in Figure 4 of Wen (2003). As Wen noted, such a system would enter the aLIGO band with a large eccentricity, $e_{1} \approx 0.9$. This occurs because GW radiation dominates within one Kozai cycle and SP is negligible. In reality, such initial conditions correspond to a highly unstable system and no merger occurs when the system is evolved in time by using the direct integrator. In fact, according to Equation (16), the quadrupole level secular equations of motion should, and our numerical integrations show that they do, result in a very small residual eccentricity for all stable configurations. In contrast the results of the direct 3-body calculations give large residual eccentricities; a few binaries enter the aLIGO band with extremely large eccentricities, $e_{1} \gtrsim 0.9$.

We investigate the importance of the octupole level terms in the lower panel of Figure 1 where we set $m_{0}=$ $8 \mathrm{M}_{\odot}, m_{1}=12 \mathrm{M}_{\odot}$ and $m_{2}=10 \mathrm{M}_{\odot}$. In this case there is a non zero octupole order perturbation which changes the rate of precession slightly, and causes variation in the outer perturber orbital eccentricity.

When expanded to the quadrupole order only, the averaged perturbing potential is axisymmetric (even for an eccentric external orbit, e.g., Katz et al. 2011). A direct consequence of this is that the magnitude of the inner orbit's angular momentum has a well defined lower bound which under the test particle approximation is simply the Kozai constant, i.e. $\ell_{1} \leq \ell_{z}=\sqrt{1-e_{1}^{2}} \cos (I)$, with $z$ chosen along the direction of $\mathbf{L}_{2}$. At the octupole

the particle positions and velocities by adopting the relative radial expressions given in Equations $(3.6 a)$ and $(3.6 \quad b)$ of Damour \& Deruelle (1985).

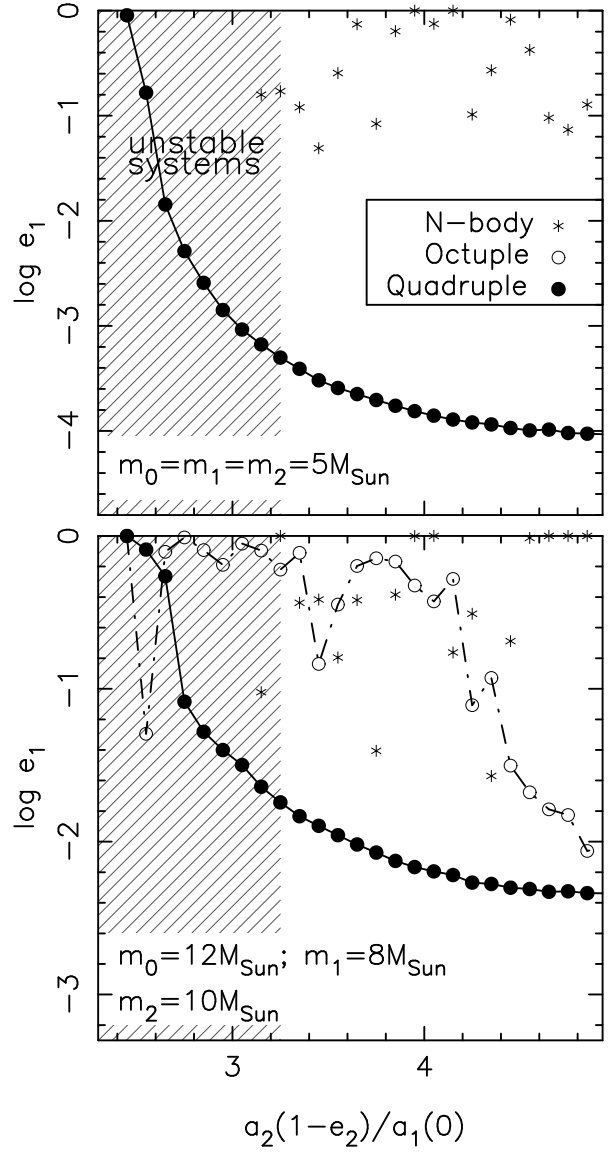

FIG. 1. - The eccentricity at the moment the inner $\mathrm{BH}$ binary enters the sensitivity window of planned ground-based interferometers as a function of the periapsis distance of the outer body, $a_{2}\left(1-e_{2}\right)$. Inside the hatched regions triple systems are unstable according to Equation [11]; our $N$-body experiments verify the accuracy of this stability criterion. In these integrations we fixed the initial inner and outer orbit semi-major axes, while we distributed the external orbit eccentricity uniformly within the range $e_{2}=(0.51,0.01)$, corresponding to the interval of periapsis distances $a_{2}\left(1-e_{2}\right) / a_{1}(0)=(2.45,4.95) \mathrm{AU}$. We randomly chose the initial true anomalies, set $\omega_{1}=\omega_{2}=0$ and $I=99^{\circ}$. In the upper panel, we set $a_{1}=2 \mathrm{AU}, a_{2}=5 \times a_{1}$. Since the inner binary components in these systems have equal masses the octupole order terms in the secular equations of motion go to zero. The corresponding results from the secular code provide a poor match to the results of the 3-body integrations, which systematically predict higher residual eccentricities. In the lower panel we set $a_{1}=0.2 \mathrm{AU}$ and $a_{2}=5 \times a_{1}$. In these integrations $m_{0} \neq m_{1}$, thus leading to a non-zero contribution of the octupole order terms. The results of the secular theory in this case are in better agreement with the results of the 3 -body integrations. However, while in the $N$-body runs a large fraction of binaries are exremely eccentric $\left(e_{1} \gtrsim 0.9\right)$ when the first enter the aLIGO band, the residual eccentricity of such binaries is substantially smaller $\left(e_{1} \sim 0.1\right)$ when the same initial conditions are evolved using the orbit average equations of motion (even when high order terms are included). Evidently, the standard secular theory fails in describing the dynamical evolution of these systems.

order the Hamiltonian describes a dynamical system of two degrees of freedom. Motion in this case differs in three important ways from motion in the (axisymmetric) quadrupole order potential. First, the lack of rotational symmetry implies that no component of the binary angular momentum is conserved. As a consequence of this the eccentricity of the inner orbit can reach much higher values near the maximum of a Kozai cycle (Ford et al. 


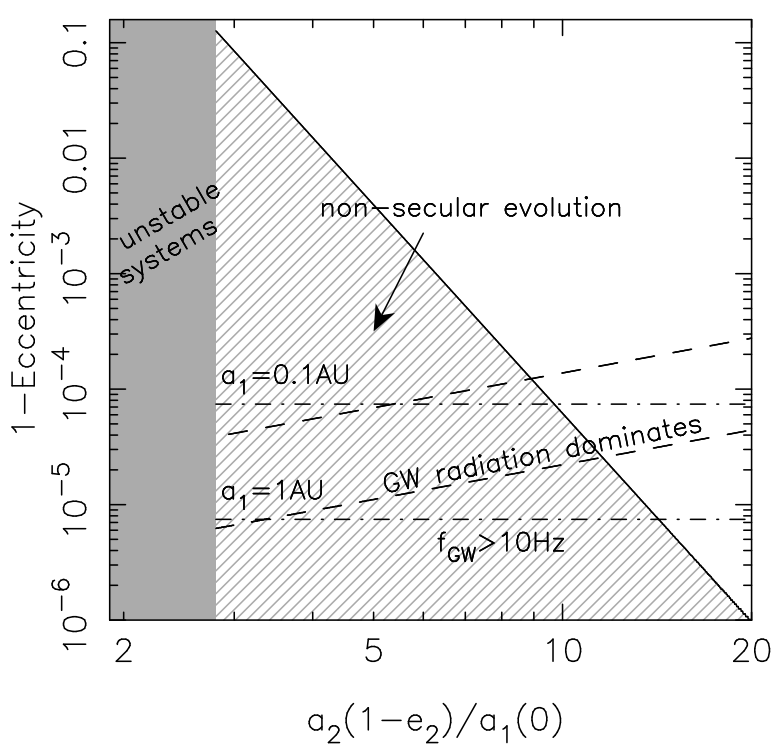

FIG. 2.- Illustrating the region of parameter space within which highly eccentric GW sources can be produced. The solid line gives the value of the critical eccentricity defined in Equation (18) as a function of the periapsis distance of the outer $\mathrm{BH}$ orbit expressed in units of $a_{1}$ and assuming $M_{b}=2 m_{2}$. Below this line a binary can reach $e_{1} \sim 1$ in a fraction of its orbital period. The gray solid area corresponds to unstable configurations according to Equation (11) where we set $e_{2}=0$ and $I=90^{\circ}$. Dashed lines enclose the region of parameter space within which GW radiation reaction dominates the evolution. These lines were computed using the approximate Equation (13) with $e_{2}=0, m_{0}=m_{1}=m_{2}=10 \mathrm{M}_{\odot}$ and $a_{1}=$ 0.1 and 1 AU. Within the dot-dashed lines the binary peak GW frequency becomes larger than $10 \mathrm{~Hz}$. Highly eccentric GW sources are produced by stable-moderately hierarchical triples with $3 \lesssim$ $a_{2}\left(1-e_{2}\right) / a_{1} \lesssim 10$. These are the only systems that can penetrate the region delimited by the hatched area in the plot before attaining $\ell_{\mathrm{GW}}$.

2000). Second, while at the quadrupole level orbits maintain their sense of rotation, in the octupole potential excursions to very high eccentricities can be accompanied by a "flip" of the orbit with respect to the direction of the total angular momentum (Naoz et al. 2011a; Lithwick \& Naoz 2011; Naoz et al. 2011b). Third, motion in the octupole potential can be stochastic, although only a restricted portion of the phase space near the libration/circulation separatrix might be expected to be chaotic (Holman et al. 1997).

Figure 1 shows that when expanded at the octupole level the secular theory leads to higher residual eccentricities and also appears to be in better agreement with the results of AR-CHAIN. This suggests that the excitation of the inner binary eccentricity seen also in the direct integrations depends on the importance of the Newtonian octupole (or even higher) order terms to the evolution which break the axisymmetric nature of the potential and lead to large eccentricities without the need of high inclination tuning.

From Figure 1]it is evident that the results of the direct 3-body integrations differ from the outcome of the secular code at least in one important way: while in the $N$-body runs for about $30 \%$ of the binaries the eccentricity remains high $\left(e_{1} \gtrsim 0.9\right)$ when they first enter the aLIGO frequency band, the residual eccentricity of such binaries is small when the same initial conditions are evolved using the orbit average equations of motion. Note also that the reliability of the secular approximation becomes pro-
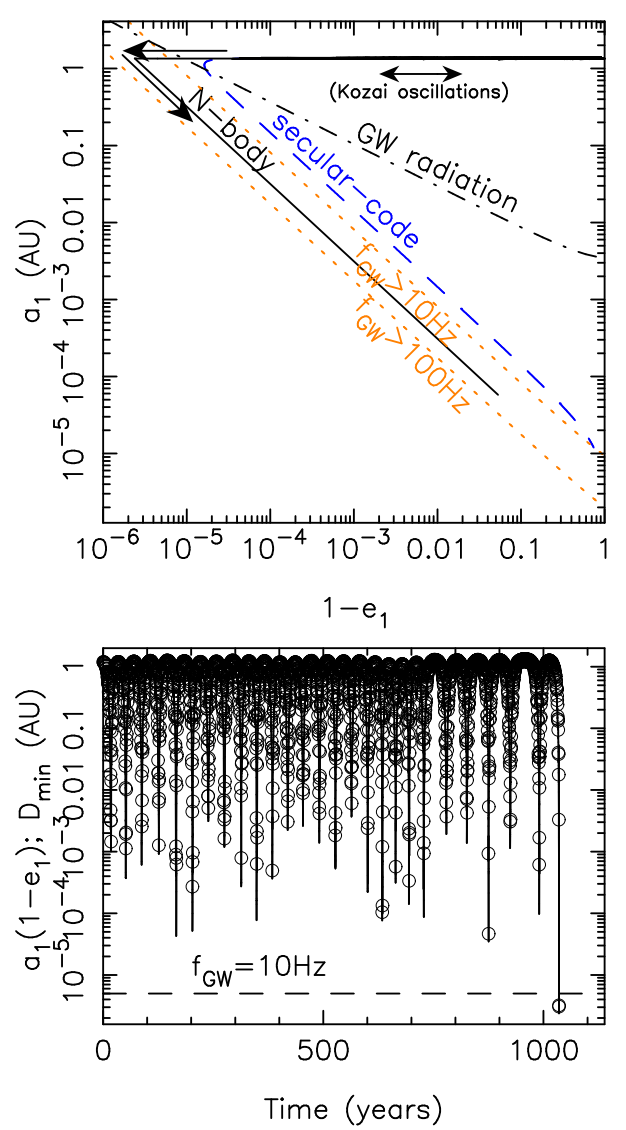

FIG. 3.- Kozai-driven evolution of a BH binary in the semi-major axis-eccentricity plane. Initial conditions are: $m_{0}=8 M_{\odot}, m_{1}=$ $12 M_{\odot}, m_{2}=10 M_{\odot} ; I=97^{\circ} ; a_{1}=1.36 \mathrm{AU}, a_{2}=6.26 \mathrm{AU}$ $e_{1}=0.01, e_{2}=0.2 ; \omega_{1}=\omega_{2}=0$. Short-dashed lines correspond to the indicated reference peak gravitational wave frequencies of 10 and $100 \mathrm{~Hz}$. The dot-dashed line in the upper panel gives the approximate condition for GW energy loss to dominate the evolution, Equation (14). To the left and below this line the BH binary eccentricity and semi-major axis rapidly decrease when the system is evolved using the double average (octupole-level) equations of motion (blue-dashed line). Arrows schematically indicate the direction in which the binary evolution proceeds. The black-solid line displays the evolution of the BH binary obtained by using more accurate 3-body integrations. After multiple periodic oscillations in the eccentricity have occurred (see lower panel), the binary reaches very high eccentricities and chirps in the aLIGO frequency band. The orbital inspiral of the BH binary in this case starts and takes place entirely within the aLIGO band. The lower panel shows time evolution of the actual distance of closest approach, $D_{\text {min }}$, between the inner binary components (open circles) and the periapsis distance, $a_{1}\left(1-e_{1}\right)$, calculated from the current value of the osculating orbital elements (solid line). Near the end of the simulation the periapsis distance between the $\mathrm{BHs}$ changes such that the GW signal enters the aLIGO band in one orbital period before the rapid orbital circularization due to GW can start. This large angular momentum change, achieved in one orbital period, allows the BHs to attain a close approach and the GW signal to enter the aLIGO band at high eccentricity. The peak GW frequency at the moment the insipral phase begins is significantly larger than $10 \mathrm{~Hz}$, about $45 \mathrm{~Hz}$ in this case.

gressively worse as $e_{2}$ (or equivalently, the contribution of the octupole order terms) approaches zero.

The discrepancy arises because, near a minimum in $\ell_{1}$, the time

$$
\left|\frac{1}{\ell_{1}} \frac{d \ell_{1}}{d t}\right|^{-1} \approx T_{\mathrm{b}} \frac{1}{5 \pi} \frac{M_{b}}{m_{2}}\left[\frac{a_{2}\left(1-e_{2}\right)}{a_{1}}\right]^{3} \sqrt{1-e_{1}}
$$

for the orbit to change from its current value of $\ell_{1}$ to 
$\ell_{1} \sim 0$ becomes shorter than the binary orbital period, $T_{\mathrm{b}}=2 \pi \sqrt{a_{1}^{3} /\left(G M_{b}\right)}$. In deriving Equation (17) we have assumed a fixed outer perturber, and adopted the instantaneous quadrupole order torque (e.g., Katz \& Dong 2013) taking the relevant limit $e_{1} \rightarrow 1$ and assuming maximal torque at the periapsis approach. This condition can then be expressed in terms of the system semimajor axes and eccentricities as:

$$
\begin{gathered}
\sqrt{1-e_{1}} \lesssim 5 \pi \frac{m_{2}}{M_{b}}\left[\frac{a_{1}}{a_{2}\left(1-e_{2}\right)}\right]^{3}= \\
8 \times 10^{-3}\left(2 \frac{m_{2}}{M_{b}}\right)\left[10 \frac{a_{1}}{a_{2}\left(1-e_{2}\right)}\right]^{3} .
\end{gathered}
$$

If the binary angular momentum becomes smaller than this critical value the orbit can evolve substantially and reach $e_{1} \sim 1$ on a timescale short compared to the binary orbital period, implying that the orbit average approximation formally breaks down. For objects of comparable masses what determines whether or not this condition is satisfied is the value of the ratio $a_{2}\left(1-e_{2}\right) / a_{1}$ in Equation (18) which parametrizes the size of the external orbit compared to the inner binary orbit. The tighter the outer orbit the larger the torque produced on the inner $\mathrm{BH}$ binary, which implies that smaller eccentricities are sufficient to meet the criterion given in Equation (18).

More specifically, what happens will depend on whether changes of the binary angular momentum occurring in one orbital period are of order or larger than the angular momentum associated with the scale at which other dynamical processes (e.g. SP or GW radiation) can affect the evolution. As an example, consider the most relevant case in which, during the high-e phase of a Kozai cycle, the binary angular momentum reaches the critical value defined in Equation (18) before SP and GW radiation terms can become important. Since the torque due to the outer $\mathrm{BH}$ is maximal near apoapsis while GW emission is peaked at periapsis, it is possible that a large change of angular momentum attained near apoapsis reduces $\ell_{1}<\ell_{\mathrm{GW}}$, and does this in a fraction of the orbital period before relativistic terms can affect the evolution. If $\ell_{\mathrm{GW}}$ is crossed while the $\mathrm{BHs}$ are near apoapsis, and the binary attains periapsis before $\ell_{\mathrm{GW}}$ is passed over, the value of the angular momentum at which energy dissipation due to GW emission becomes important can be significantly smaller than $\ell_{\mathrm{GW}}$. This allows the binary to have large eccentricities at large GW frequencies. Figure 2 illustrates the region of parameter space inside which binaries can undergo the non-secular evolution discussed above and attain very large eccentricities before GW radiation dominates the evolution. These binaries reside in highly inclined-moderately hierarchical configurations with $3 \lesssim a_{2}\left(1-e_{2}\right) / a_{1} \lesssim 10$.

An example is given in Figure [3] which displays the time evolution of semi-major axis and eccentricity of a $\mathrm{BH}$ binary merger driven by the Kozai mechanism. Toward the end of the simulation, the binary achieves a large enough change in angular momentum such that its peak GW frequency crosses the aLIGO frequency band in a fraction of an orbital period. Encounters occurring before this time are at large enough distance that dissipative and non-dissipative relativistic corrections are both negligible. The semi-major axis of the binary remains ap- proximately unchanged during the evolution before GW radiation initiates the inspiral. In the case shown the inspiral of the BH binary due to GW energy loss starts when its peak GW is about $45 \mathrm{~Hz}$, or, equivalently, when the periapsis distance is approximately $10 M_{b}$ in geometric units, and therefore takes place entirely within the $10 \mathrm{~Hz}$ frequency band. In contrast, when using the double average equations of motion, which do not contain information about orbital phases, the maximum eccentricity attainable by the $\mathrm{BH}$ binary is artificially limited by GW energy loss near $\ell_{\mathrm{GW}}$.

The evolution of the GW signal emitted by highly eccentric binaries in the aLIGO frequency band was discussed before in O'Leary et al. (2009) and Kocsis \& Levin (2012). The eccentric signature of these binaries would make them distinguishable from other GW sources; their GW signal will consist of an initial phase of repeated bursts for minutes to days emitted during periapsis approach where the GW emission is maximized, followed by a continuous chirp signal and an eccentric merger. The relevant timescales that determine the GW waveform are the orbital time, $\Delta t_{\text {orb }}=$ $2 \pi \sqrt{a_{1}^{3} / G M_{b}}$, and the duration of the periapsis passage, $\Delta t_{\text {per }}=\pi \sqrt{\left[a_{1}\left(1-e_{1}\right)\right]^{3} / G M_{b}}$. The characteristic duration of the GW bursts is $\Delta t_{\text {per }}$, while $\Delta t_{\text {orb }}$ is the time between two consecutive bursts. For $\Delta t_{\text {per }} \ll \Delta t_{\text {orb }}$, the waveform consists of a train of GW bursts of characteristic duration $\Delta t_{\text {per }}$ emitted quasi-periodically every $\Delta t_{\text {orb }}$. Later, when $\Delta t_{\text {per }} \sim \Delta t_{\text {orb }}$, the signal becomes continuous in time domain. For the system of Figure 3 we find $\Delta t_{\text {orb }}=1.8 \times 10^{5}$ and $\Delta t_{\text {per }}=3.4 \times 10^{-4}$ minutes at the instant where the GW-driven inspiral of the inner binary begins, and $\Delta t_{\text {orb }}=5.1 \times 10^{-2}$ and $\Delta t_{\text {per }}=3.1 \times 10^{-4}$ minutes when the binary eccentricity has decreased to $e_{1}=0.95$.

\section{COALESCING BLACK HOLE BINARIES IN GLOBULAR CLUSTERS}

Mergers of $\mathrm{BH}$ binaries forming via dynamical interactions in GCs might dominate the total $\mathrm{BH}-\mathrm{BH}$ merger event rates for aLIGO and are therefore of wide astrophysical interest (Portegies Zwart \& McMillan 2000; Abadie et al. 2010). Here, we determine the type and properties of $\mathrm{BH}$ binary mergers in GCs induced by a combination of Kozai cycles and GW energy loss. Similar studies were previously conducted by Miller \& Hamilton (2002a), Wen (2003) and O'Leary et al. (2006). These papers, however, were limited to the traditional orbit average secular theory which we have shown to be inaccurate in describing the evolution of such systems. To overcome the limitations of the orbit average approach we perform in what follows direct integrations of the PN equations of motion describing hierarchical BH triples.

\subsection{Initial setup}

The simulation initial conditions were generated as detailed below. The most important quantities that affect the maximum eccentricity a system can reach (and consequently its merger time) are $a_{1}$ and $a_{2} / a_{1}$. For example, the details of the mass parameter distributions have a relatively little effect on the eccentricity a system can reach. Monte Carlo simulations suggest a distribution of periods for $\mathrm{BH}$ binaries inside globular clusters 
which is approximately flat in log space (e.g., Figure 6 of Downing et al. 2010). Accordingly, in a first set of simulations (MOD1 hereafter) the initial BH binary semimajor axis, $a_{1}$, was drown from a distribution which is flat in $\log$ space, $f(\log p) \propto c$ const, which corresponds to $f(a) \propto 1 / a$ (i.e., Öpik's law). The lower and upper limits for $a_{1}$ were $0.2 \mathrm{AU}$ and $30 \mathrm{AU}$ respectively. The lower limit of $0.2 \mathrm{AU}$ corresponds roughly to the minimum value of the semi-major axis distribution of $\mathrm{BH}$ binaries obtained via Monte Carlo simulations in Downing et al. (2010); the upper limit of $30 \mathrm{AU}$ is the same adopted in Wen (2003). The outer orbit semi-maor axis, $a_{2}$, was also log-uniformly distributed. Thus we assumed that the orbital semi-maor axis of the third component in a given triple could be chosen from a similar distribution of the parent binaries. We imposed an upper limit of $a_{2}=30 \times a_{1}$, given that the parameter range for $a_{1}$ decreases with increasing $a_{2} / a_{1}$ (Blaes et al. 2002; Wen 2003): for $a_{2} / a_{1}>30$ the binary merger time (Kozai timescale) becomes typically much longer than the collision time with other field stars (Equation 20] below). The initial eccentricities, $e_{1}$ and $e_{2}$, were sampled from a thermal distribution, $N(e) \propto e$. The physics behind such $e$ and $a$ distributions is well known: it is the results of energy relaxation in few body gravitational interactions which affect the binary/triple dynamical evolution.

We adopted a random distribution in $\omega_{1}$ and $\omega_{2}$ and random in $\cos (I)$ with $85^{\circ} \leq I \leq 110^{\circ}$, as required to obtain extremely large eccentricities. Orbital phases were also randomly distributed. Since unstable triples are likely to dissociate before completing one Kozai cycle (e.g., Figure 11), we only considered configuratios which satisfied the stability criterion given by Equation (11).

We sampled the $\mathrm{BH}$ masses from the exponential distribution

$$
N(m)=\frac{\exp \left(M_{c} / M_{0}\right)}{M_{0}} \exp \left(-m / M_{0}\right)
$$

for $m \geq M_{c}$ and $N(m)=0$ otherwise. This choice of the mass distribution is motivated by theoretical expectations based on the energetics and dynamics of supernova explosions (Frver \& Kalogera 2001). The values of the mass scale in the exponential and the cutoff mass are respectively $M_{0}=1.2 \mathrm{M}_{\odot}$ and $M_{c}=$ $6.32 \mathrm{M}_{\odot}$ (Ozel et al. 2010). This choice of parameters is consistent with constraints from observations of Galactic soft X-ray transients which indicate a significant paucity of BHs with masses less than $\sim 5 \mathrm{M}_{\odot}$ (Bailyn et al. 1998; Ozel et al. 2010; Farr et al. 2011).

Although the distributions assumed above are quite reasonable, we stress that exhaustive studies have not been made for the results of binary-binary interactions, and information about whether the stable hierarchical triples that result have $a_{1}, e_{1}, a_{2}$, and $e_{2}$ distributions that are similar to the distributions of the parent binaries remains unclear. This might therefore represent a source of uncertainty for our study. In order to understand the dependence of our results on the assumed initial conditions, we perform an additional set of simulations (MOD2 hereafter) where we adopted a uniform distribution in both $a_{1}$ and $a_{2}$, a uniform distribution in eccentricities and set $m_{0}=m_{1}=m_{2}=10 \mathrm{M}_{\odot}$. Other parameters and limits of the various distributions were the same as defined above. This second set of initial conditions contain a larger number of wider configurations with larger outer periapsis separation with respect to MOD1; on the basis of our previous analysis (e.g., Figure 21) we would therefore expect the secular orbit average code to provide a better description of the $\mathrm{BH}$ triple dynamics in this case and the coalescing binaries to have smaller eccentricities in the aLIGO band. MOD2 corresponds to our most conservative assumptions.

Due to the crowded stellar environment of GCs, triple systems might be perturbed through encounters with other stars on timescales that are shorter than the relevant secular timescale (Kozai evolution). Such encounters will alter the orbital properties of the triple significantly or even disrupt it. To account for this we set the final integration time in our simulations equal to the timescale,

$$
T_{\text {coll }}=2 \times 10^{5} \operatorname{yr}\left(\frac{10^{6} \mathrm{pc}^{-3}}{n}\right)\left(\frac{\mathrm{AU}}{a_{2}}\right)\left(\frac{30 \mathrm{M}_{\odot}}{M_{b}+m_{2}}\right),
$$

for collisions with field stars (Binney \& Tremaine 1987), where $n=10^{6} \mathrm{pc}^{-3}$ is the number density of stars in the GC (e.g., Miller \& Hamilton 2002a; Wen 2003). We first evolved the systems forward in time by using the octupole level orbit average equations of motion and obtained an estimate of the binary merger time, $T_{\mathrm{AV}}$. We discarded systems that after a time $T_{\text {coll }}$ did not produce a merger event, while initial conditions that successfully lead to a merger, i.e.,

$$
T_{\mathrm{AV}}<T_{\text {coll }}
$$

were realized as point-mass particle representations and evolved forward in time by using AR-CHAIN. The maximum time of integration in the 3-body runs was also set to $T_{\text {coll }}$.

Strictly speaking, since the orbit averaged approach is inaccurate in determining the merger properties of the triple, selecting systems on the basis of Equation (21), although computationally convenient, might introduce some artificial bias in our results. To check on this, we run an additional set of direct simulations (MOD1-bis hereafter) in which we only consider systems which satisfy the condition $T_{\mathrm{NB}}<T_{\text {coll }}$, with $T_{\mathrm{NB}}$ the merger time of individual systems computed via direct 3 -body integrations. Thus, the initial conditions for the 3-body integretions in MOD1-bis were not selected on the basis of Equation (21), rather they were sampled from the entire distribution corresponding to MOD1, and directly integrated with AR-CHAIN until either the inner binary merged, the triple was dissociated, or the maximum time, $T_{\text {coll }}$, was reached. As shown in the analysis that follows, the correspondence between the results of MOD1 and MOD1-bis is good, assuring us that general conclusions of our paper are indeed not particularly sensitive to the assumed initial conditions and/or methodology adopted to generate the initial conditions. We stress, however, that given the idealized initial conditions used in this paper and the uncertainty in the properties of $\mathrm{BH}$ triple systems in GCs our results should be only considered as a set of baselines for making predictions about the GW signal produced by such systems. In total, we run 3000 

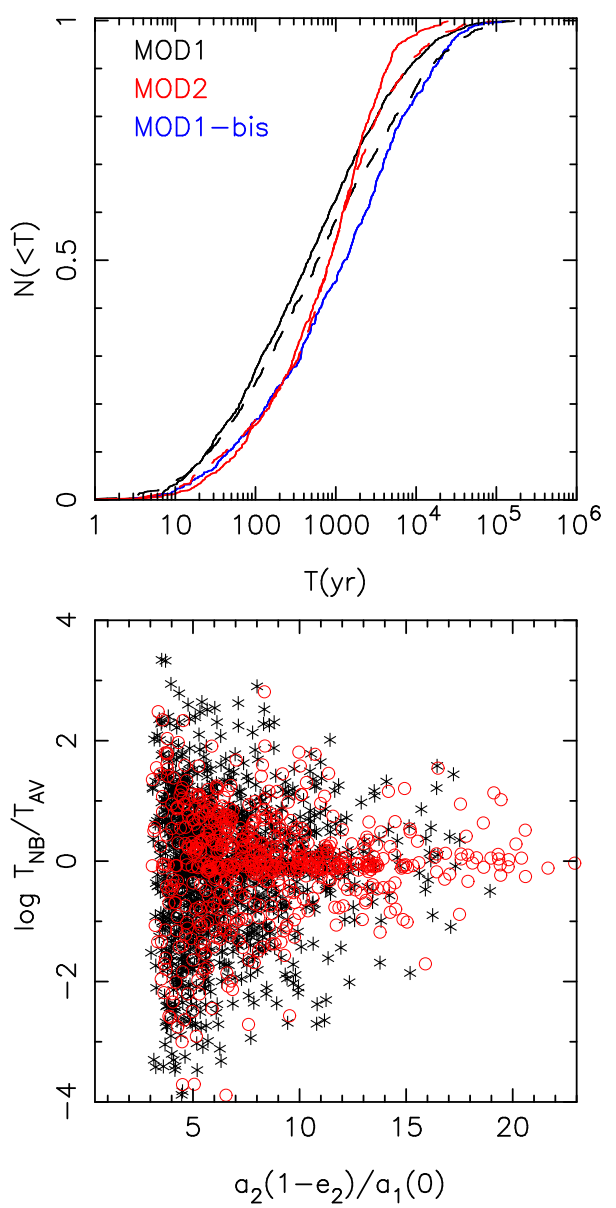

FIG. 4.- Top panel shows the cumulative distribution of merger times obtained via direct numerical integration of the equations of motion (solid lines) and from the orbit average Hamiltonian model (dashed lines). Lower panel gives the actual merger time of individual systems $\left(T_{\mathrm{NB}}\right)$ divided by the same timescale when computed by using the secular orbit average code $\left(T_{\mathrm{AV}}\right)$, as a function of the periapsis separation of the outer $\mathrm{BH}$.

3-body simulations.

\subsection{Results}

\subsubsection{Merger time distributions}

In MOD1 we found that $61 \%$ of systems that merged when evolved using the double average equations of motion also merged when evolved via direct $N$-body integrations. In MOD2 this percentage increases, but only slightly, to $70 \%$. The upper panel of Figure 4 compares the cumulative distribution of merger times for BH binaries calculated via direct integrations with merger times obtained by using the orbit average code. The two methods generate similar merger time distributions.

The bottom panel in Figure 4 shows the merger time of individual systems, $T_{\mathrm{NB}}$, which we divided by the merger time computed by using the orbit average model and plotted as a function of $a_{2}\left(1-e_{2}\right) / a_{1}$. Most mergers occur at $3 \lesssim a_{2}\left(1-e_{2}\right) / a_{1} \lesssim 10$, a regime where the assumptions behind the secular approach are not valid. As a result the merger time obtained via the standard Kozai treatment can be inaccurate by several orders of magnitude; this is especially true for the systems with the shortest periapsis separations. In MOD1 (MOD2) we found that $13 \%(10 \%)$ of binaries have $T_{\mathrm{NB}} / T_{\mathrm{AV}}>10$ and $9 \%(10 \%)$ have $T_{\mathrm{NB}} / T_{\mathrm{AV}}<0.1$. These results suggest that the orbit average Hamiltonian model can lead to misleading results and to an incorrect determination of the merger time of BH binary mergers in GCs (a point we return to in $\S$ 或).

The merger time distribution of MOD1-bis is slightly biased towards longer merger times when compared to the results of the orbit average code. On the basis of our previous analysis, this is indeed not surprising. As described in the previous section the orbit average approximation becomes unreliable if the angular momentum of the inner binary changes on a time which is comparable or shorter than the inner binary orbital period, $T_{\mathrm{b}}$. In the orbit average integrations systems that satisfy such condition typically merge when they first enter the high eccentricty phase of a Kozai cycle. Thus, the typical merger timescale of these systems will be the time scale to reach the maximum eccentricity of a Kozai cycle, i.e. roughly the Kozai time scale. In the N-body integrations instead, the binary angular momentum can reach and pass over the $\ell_{1}<\ell_{\mathrm{GW}}$ region before the binary attains periapsis. If this is the case, it might take many Kozai cycles before the two $\mathrm{BHs}$ attain a periapsis distance such that the GW radiation dominated phase can start.

\subsubsection{Eccentricity distributions}

The left and right panels of Figure 5 give the number and cumulative distributions of eccentricities for MOD1 at the moment the GW signal enters the $10 \mathrm{~Hz}$ and the $40 \mathrm{~Hz}$ frequency bands respectively. The histograms are normalized to the total number of systems that manage to merge before being significantly perturbed by encounters with field stars. MOD1 and MOD1-bis produce similar residual eccentricity distributions with about $50 \%$ (20\%) of merging systems with eccentricities larger than 0.1 and roughly $10 \%(5 \%)$ of them with extremely high eccentricities, $1-e_{1} \lesssim 10^{-5}$, at the moment they enter the $10 \mathrm{~Hz}(40 \mathrm{~Hz})$ frequency band. The reason for the gap in the eccentricity distributions between $e_{1} \sim 0.6$ and 1 resides in the fact that when a $\mathrm{BH}$ binary inspiral begins outside the $10 \mathrm{~Hz}$ requency then it remains outside this frequency band as it circularizes and evolves at roughly constan periapsis and peak gravitational wave frequency (e.g., dashed blue line in Fig. $3)$. Thus, even an initially very eccentric binary will be typically circularized by the time it reaches the $10 \mathrm{~Hz}$ frequency.

Results of integrations of MOD2, in which eccentricities and semi-major axes follow uniform distributions, are displayed in Figure 6. In this case we found that roughly $30 \%(10 \%)$ of merging systems have eccentricities larger than 0.1 and $7 \%(3 \%)$ of them have extremely high eccentricities, $1-e_{1} \lesssim 10^{-4}$, at the moment they enter the $10 \mathrm{~Hz}(40 \mathrm{~Hz})$ frequency band. Due to the larger values of $a_{2}\left(1-e_{2}\right) / a_{1}$ adopted in this second set of initial conditions the residual eccentricity distributions contain less eccentric orbits than in Figure 5, in agreement with the results of $\S 3.2$. However, the basic results of our study, i.e., a large fraction of coalescing binaries with a finite eccentricity and about $10 \%$ of them with an extreme eccentricity, remain essentially unchanged. After this paper was submitted, Antognini et al. (2013) pre- 

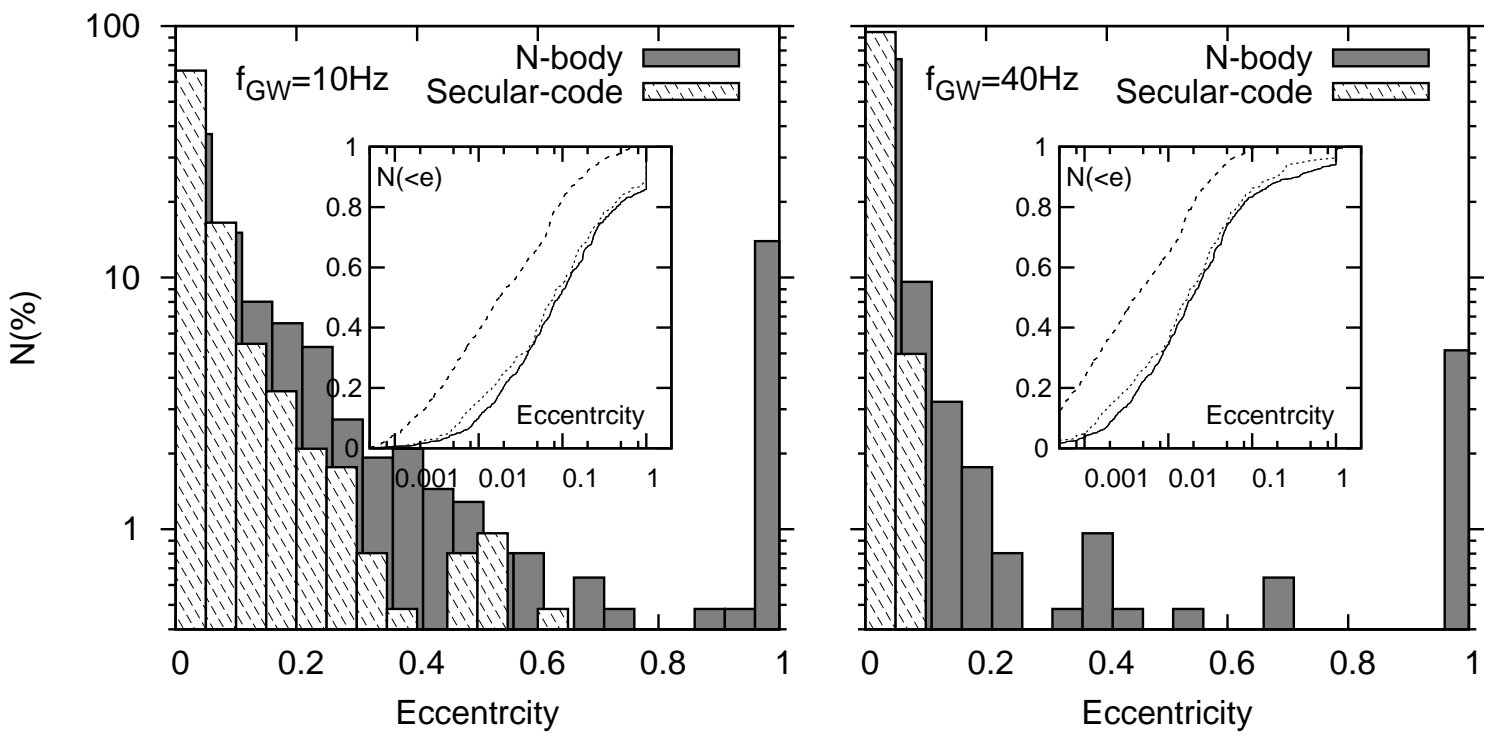

FIG. 5.- Histograms of the eccentricity distribution in MOD1 of coalescing BH binaries driven by the Kozai mechanism in GCs when their emitted GW signal first enters the $10 \mathrm{~Hz}$ (left panel) and the $40 \mathrm{~Hz}$ (right panel) frequency bands. Insert panels show the corresponding cumulative distribution of eccentricities. Distributions are normalized to the total number of systems that merge before being perturbed by encounters with other stars in the cluster. Systems were evolved using both the octupole-order orbit average equations of motion (hatched histograms and dashed lines) and more accurate 3-body direct integrations (filled histograms and solid lines). The 3-body runs produce orbital distributions that are significantly more biased towards large eccentricities, and predict that about $50 \%$ of merging binaries have eccentricities larger than 0.1 and that $10 \%$ of them posses extremely large eccentricities $\left(e_{1} \sim 1\right)$ when they first enter the aLIGO frequency band. The number of these highly eccentric GW sources is strongly underestimated when using the orbit average (secular) code. Dotted lines in the insert panels give the cumulative distribution of eccentricities of coalescing BH binaries in MOD1-bis. In this model the 3-body initial conditions were sampled from the entire distribution corresponding to MOD1, instead of being selected on the basis of Equation (21).
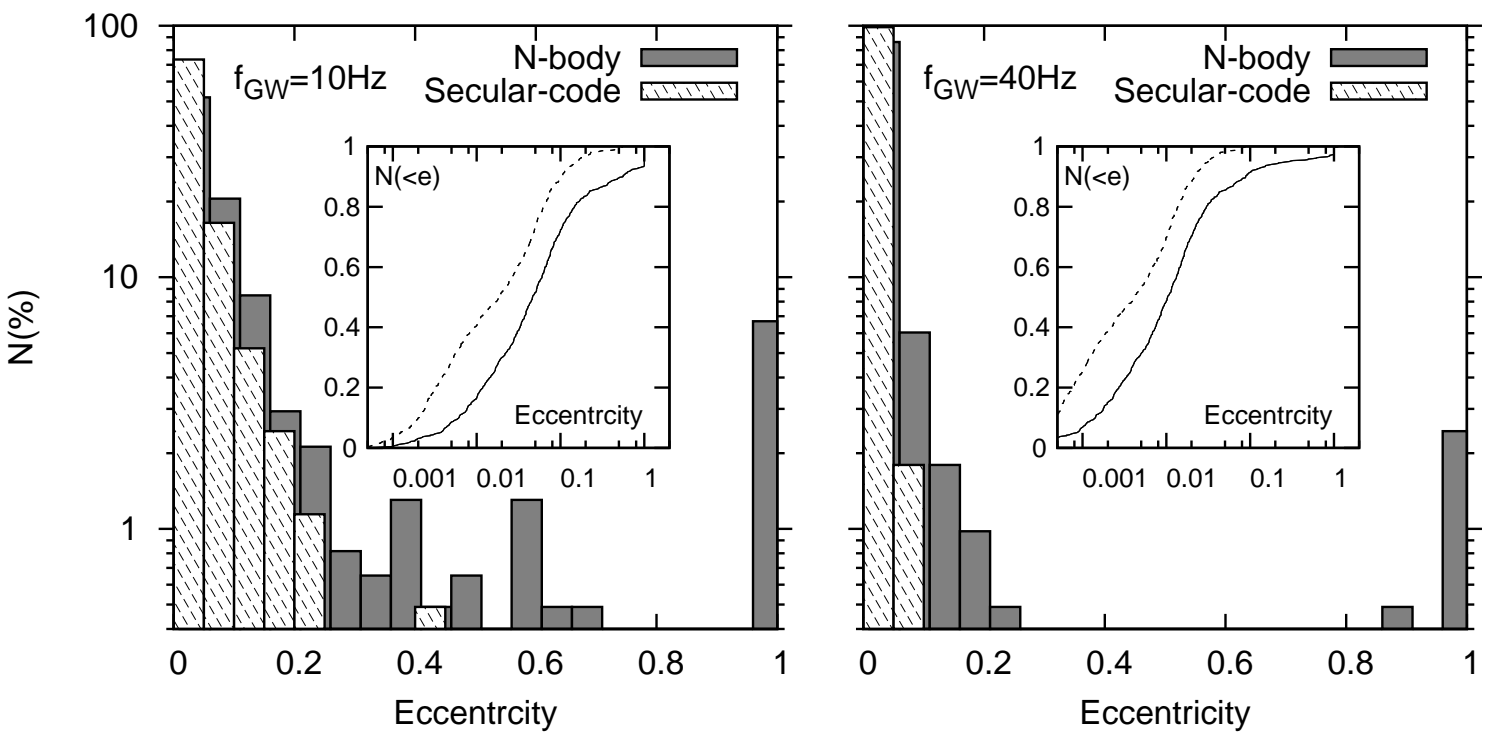

FIG. 6.- Same as Figure 5 but adopting an initial uniform distribution in semi-major axes and eccentricities (MOD2).

sented a similar study on the breakdown of the secular approximation and found a similar fraction of eccentric compact object binary mergers.

The initial conditions for MOD2 are somewhat more similar to what was adopted in Wen (2003). Using the quadrupole order orbit average equations, Wen finds that about $2 \%$ of binaries have $e_{1} \sim 1$ at $10 \mathrm{~Hz}$. When using the double average equations of motion, we find, contrary to Wen, that the percentage of such high eccentric sources is only $0.1 \%$ of the total number of coalescing binaries.

We believe that the nature of the discrepancy resides at least partially in the criterion used in Wen (2003) for selecting stable versus unstable systems, see Equation (42) of Wen (2003). This equation ignores the $\left(1-e_{2}\right)$ term that appears at the denominator in the right hand side of our Equation (11). This could allow for initial conditions with larger values of $e_{2}$ and therefore smaller periapsis separation than in our simulations and lead to an artificial bias toward large residual eccentricities as shown in Figure 1. We already mentioned, as an example, the system of Figure 8 of Wen (2003). This system produces a very eccentric GW source, $e_{1} \sim 0.9$ at $10 \mathrm{~Hz}$, but in reality this is a highly unstable triple according to the 


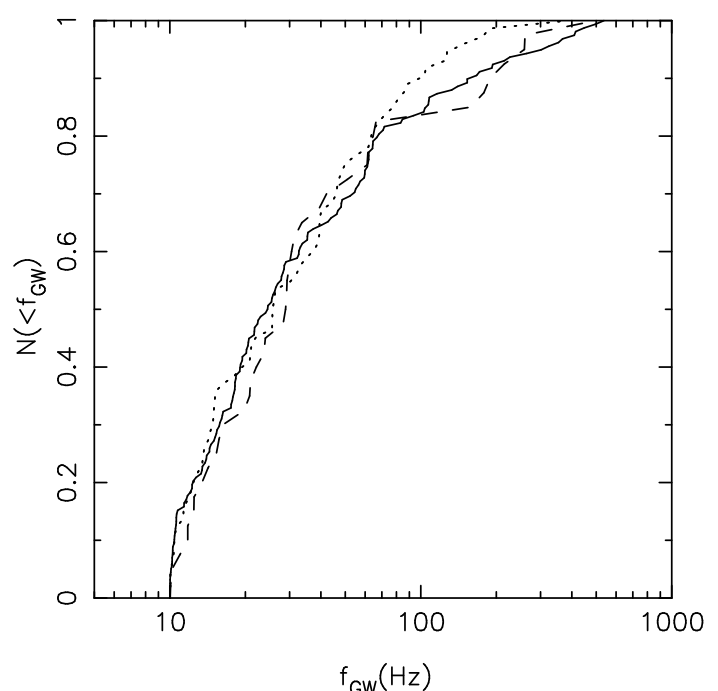

FIG. 7.- Dominant GW frequency at the instant where the GWdriven inspiral of inner binary begins to dominate over the Kozaidynamics. Shown is a subset of the 3-body configurations from Figure 5 (solid line for MOD1 and dotted line for MOD1-bis) and Figure 6 (dashed line), namely those with $e_{1} \geq 0.9$ at this instant. $80 \%$ of these binaries reach this point at a GW frequency of $f_{\mathrm{GW}} \leq$ $100 \mathrm{~Hz}$.

Mardling \& Aarseth (2001) stability criterion (see also our Figure 10. Moreover, we note that most of the initial conditions that in Wen's Monte-Carlo experiments would allow the binary to enter the aLIGO band at high eccentricities, $a_{2} / a_{1}=3$ in her Table 1 , are unstable even according to Equation (42) of Wen. Additional experiments, in which we varied integration parameters, changed the integrator itself, and also used a different code (that of Prodan \& Murray 2012) convinced us of the robustness of our results.

The dominant GW frequency at which a binary first becomes a potentially detectable GW source can be substantially larger than $10 \mathrm{~Hz}$ (e.g., Figure 3). This could in principle affect the detectability of eccentric sources even assuming one has a perfect inspiral waveform template. In fact, the detectability of a $\mathrm{BH}$ binary is related to how much energy is radiated in the frequency range where aLIGO will be more sensitive, i.e. around $100 \mathrm{~Hz}$. If the pericenter of an highly eccentric orbit is such that it has a peak frequency above this range, then most of the GW energy would be radiated at higher frequencies than $100 \mathrm{~Hz}$, making such source difficult to detect. The typical GW peak frequency of eccentric sources is given in Figure 7 which displays the cumulative distribution of peak GW frequencies at the moment the inspiral due to GW energy loss starts. We do this only for systems in the left panel of Figure 5and Figure 6 with residual eccentricities larger than 0.9. We find that about $80 \%$ (50\%) of coalescing binaries have $f_{\mathrm{GW}}<100 \mathrm{~Hz}\left(f_{\mathrm{GW}}<40 \mathrm{~Hz}\right)$ at the instant where the GW-driven inspiral begins to dominate over the Kozai-dynamics. Thus most eccentric sources could be efficiently detected by aLIGO, provided an efficient search strategy.

\section{DISCUSSION AND CONCLUSIONS}

We have explored the dynamical evolution of hierarchical $\mathrm{BH}$ triples and investigated the conditions which lead to the rapid merger of $\mathrm{BH}$ binaries in GCs. We did this by using the direct numerical integrator ARCHAIN which includes PN corrections to the equations of motion up to order 2.5 and the algorithmically regularized chain structure to avoid singularities (Mikkola \& Merritt 2008). We have shown that the presence of a third outer $\mathrm{BH}$ can drive the inner binary at very high eccentricities; at which point the $\mathrm{BHs}$ can rapidly merge before the triple system is perturbed or disrupted through gravitational encounters with cluster field stars and on timescales much shorter than for similar binaries that are evolved in isolation. We determined the properties of coalescing $\mathrm{BH}$ binaries in GCs and compared the results of our 3-body simulations to the predictions of the standard orbit average treatment in which the equations of motion are averaged over the rapidly varying mean anomalies of inner and outer orbit. We have demonstrated that the orbit average treatment leads to incorrect results if the inner binary is orbited by an outer perturber at a small distance (Equation [18).

The implications of our results are discussed in what follows.

\subsection{Eccentric gravitational wave sources}

The 3-body integrations presented in this paper predict a large number $(30-50 \%)$ of GW sources with a substantial eccentricity $\left(e_{1} \gtrsim 0.1\right)$ in the high frequency band of GW detectors. We predict the existence of a large population of extremely eccentric GW sources, $\left(1-e_{1}\right) \lesssim 10^{-5}$, that will be potentially detectable by advanced GW detectors. These mergers are driven by Kozai (like) dynamics in moderately hierarchical triple systems that are predicted to efficiently form via dynamical exchange interactions in the dens core of globular clusters.The number of these highly eccentric GW sources is strongly underestimated when adopting the secular approach.

Eccentric sources have a unique GW signal compared to circular binaries. Initially, they will repeatedly burst in the aLIGO band at each periapsis approach where the GW emission is maximized. The broad-band nature of the GW signal emitted during the repeated bursts phase could make these sources potentially detectable at larger distances and in a broader mass range than circular mergers (e.g., O'Leary et al. 2009; Kocsis \& Levin 2012). For example, the inspiral of a stellar BH with another $\mathrm{BH}$ of mass $\sim 10^{3} \mathrm{M}_{\odot}$ may be revealed by aLIGO only if the event is sufficiently eccentric during plunge (O'Leary et al. 2009). Later the binary transits to lower eccentricities and to a continuous powerful chirp signal within the frequency band of aLIGO type detectors.

The detection of GW signal requires a bank of theoretical modeled binary inspiral waveforms as filters. Current searches for binary BHs rely on circular binary templates based on PN modeling of the inspiral phase in the weak-field slow-motion regime (Blanchet 2006), and numerical relativity simulations to describe the merger phase in the strong gravity field regime (Pretorius 2005; Campanelli et al. 2006; Baker et al. 2006). An extensive review of the current status of numerical simulations of compact binary mergers is given in Pfeiffer (2012). There are at least three reasons why filter templates are currently limited to circular binary waveforms. First, circular binaries remain a very important GW source, prob- 
ably the most important one. Specifically, NS-NS systems are the primary target for aLIGO. Second, for circular binaries, one can push the PN expansions to higher order (via energy balance arguments). This allows one to constract PN waveforms that are of sufficient quality for detection for total mass $m_{0}+m_{1} \leq 12 \mathrm{M}_{\odot}$. Currently, eccentric PN waveforms are not known to similar high order, thus an eccentric PN waveform of sufficient accuracy does not exist. Third, circular binaries tend to be dominated by $(2,2)$ and $(2,-2)$ radiation. If one discards all other modes, and looks only for $(2,2)$ waveform-modes, then it turns out sky-position, source-orientation, and orbital phase of the binary are all degenerate. Therefore, the dimensionality of the parameter space dramatically collapses. Eccentric waveforms will have a much more complex emission pattern, breaking this degeneracy. Adding eccentricity to binary templates would also imply that more templates are needed which will require a higher signal to noise threshold for a given significance. This might reduce, at least in part, the detectability benefit one could get for eccentric binaries.

The effect of eccentricity in aLIGO searches was investigated in Brown \& Zimmernman (2010) and more recently in Huerta \& Brown (2013). These authors find that circularized waveforms are sufficient to recover the signal emitted by eccentric sources with eccentricities less than $\sim 0.1$ at a fiducial GW frequency of $14 \mathrm{~Hz}$. Thus the eccentric binaries found in our study cannot be efficiently detected by advanced detectors with current search pipelines. Optimal searches for such systems would require replacement of the conventional quasi-circular aLIGO templates with eccentric-binary templates for data analysis, or the use of more practical excess power searches with stacking (East et al. 2013).

Although eccentric binaries are frequent in our simulations, we found that about half of them will posses eccentricities smaller than 0.1 when their dominant GW frequency becomes larger than $40 \mathrm{~Hz}$ such that they might be detected even with regular templates. After detection, the careful re-analysis of the aLIGO data could reveal "pre-merger flares" from Kozai resonances. If those are found, the precise timing of the pre-merger flares would carry information about the triple system. The detection of such systems could give us unprecedented insights on the dynamical processes that have shaped the dense central environment of GCs and galaxies and lead to the formation and growth of massive and intermediate mass BHs (Miller \& Hamilton 2002b).

\subsection{Merger rates and black hole populations in globular clusters}

Adopting standard initial mass functions, about $1 \%$ of the total mass in a stellar system will be in $\mathrm{BHs}$ that formed through the supernova explosions of the most massive stars $\left(\gtrsim 20 M_{\odot}\right)$. Soon after their formation, the BHs will be the heaviest component of the stellar cluster, with a mass about 10 times larger than the mass of a typical cluster star (Woosley et al. 2002). Gravitational encounters tend to accelerate the lowest mass stars to the highest velocities, at the expense of the specific kinetic energy of the BHs, which will migrate down to the cluster core. This process, typically referred to as "masssegregation" (Spitzer 1987), leads to the formation of a centrally concentrated sub-cluster of stellar BHs and to favorable conditions for the assembly of $\mathrm{BH}$ binaries. The coalescence of such binaries through GW radiation could be a major observational target for the next generation of ground-based GW detectors (e.g., Sadowski et al. 2008).

Due to the computational challenge of simulating the long-term evolution of massive clusters with direct 3body simulations, predictions of event rates for the GW sources investigated in this paper remain largely uncertain. A key question is whether BHs are efficiently ejected through strong binary interactions during the cluster evolution. Efficient depletion of the remnant population would decrease the chance for the $\mathrm{BH}$ s to undergo repeated exchange interactions in the cluster core and become part of hierarchical triple systems. For the less massive and ancient GCs, the $\mathrm{BH}$ population might selfdeplete in less than a few Gyr before the cluster enters the cosmological volume of aLIGO $(z \sim 2)$. This will reduce the number of systems that will manage to merge within GCs due to the Kozai mechanism and become detectable sources of GW radiation.

Monte-Carlo simulations have been extensively used to study the evolution of BH populations in GCs and to determine the event rates of $\mathrm{BH}$ binary mergers in the Universe (e.g., O'Leary et al. 2006; Downing et al. 2010, 2011). These models suggest that after its formation the cluster's BH population typically evaporates over a Gyr timescale. This prediction has been challenged by recent theoretical studies (Morscher et al. 2013; Sippe \& Hurlev 2013) and by observational evidence (Maccarone et al. 2007; Brassington et al. 2010; Maccarone et al. 2011; Strader et al. 2012) which suggest that old GCs may still contain hundreds of stellar $\mathrm{BHs}$ at present. We add that most Monte-Carlo codes do not allow for the formation of the long-lived hierarchical triples investigated in this paper (e.g., Downing et al. 2010, 2011) or when they do (O'Leary et al. 2006) they employ the orbit average formalism which we have shown to lead to unreliable results and inaccurate determination of merger times (e.g., Figure 4). An isolated example of high precision $N$-body simulations of the evolution of stellar clusters containing a population of massive remnants was recently presented by Aarseth (2012). This author finds that the conditions for $\mathrm{BH}$ binary mergers in GCs are typically initiated by a combination of GW energy loss and the Kozai resonance induced by the presence of a third object, usually another $\mathrm{BH}$.

More sophisticated $N$-body simulations, or MonteCarlo codes including more precise prescriptions for the dynamical evolution of hierarchical $\mathrm{BH}$ triples would be required in order to address the role of the Kozai resonance in determining the rates of $\mathrm{BH}$ binary mergers in GCs. It is useful however to give a simple estimate of how many such eccentric events are likely to be detected with aLIGO.

We estimate the detection rate of eccentric BH binary mergers due to the Kozai mechanism as:

$$
\Gamma_{\text {aLIGO }}=\frac{4 \pi}{3} D^{3} n_{\mathrm{cl}} \Gamma_{\text {merge }} f_{\text {triple }} f_{\text {ecc }}
$$

where $D$ is the maximum distance from which the emitted GW signal from a BH binary inspiral can be detected, $n_{\mathrm{cl}}$ is the number density of GCs, $\Gamma_{\text {merg }}$ is the merger rate of $\mathrm{BH}$ binaries that occur within the cluster, $f_{\text {triple }}$ is the fraction of such mergers induced by the Kozai mecha- 
nism, and $f_{\text {ecc }}$ is the fraction of mergers due to the Kozai mechanism which retain a large eccentricity, $e>0.1$, in the aLIGO band. Based on the Monte Carlo simulations of O'Leary et al. (2006) (see their Table 2) we take a conservative rate of $\approx 10$ mergers inside GC per $10^{10} \mathrm{yr}$ and that a fraction $f_{\text {triple }} \approx 0.05$ of these mergers are due to the Kozai mechanism. From our simulations we have $f_{\text {ecc }} \approx 0.5$. If we take a globular number density of $10 / \mathrm{Mpc}^{3}$ (Brodie \& Strader 2006), and that BH-BH mergers can be seen out to a sky-averaged distance of $1 \mathrm{Gpc}$ (as expected for $10 \mathrm{M}_{\odot}-10 \mathrm{M}_{\odot}$ coalescences), and if we assume that mergers are distributed uniformly over the lifetimes of the globulars (as opposed to happening primarily early on), then Equation (22) gives an aLIGO detection rate of eccentric $\mathrm{BH}$ binary mergers due to the Kozai mechanism of $\Gamma_{\text {aLIGO }} \approx 1 / \mathrm{yr}$. Thus a uniform rate of mergers amounting to a few per Hubble time per cluster gives a relatively large population of eccentric $\mathrm{BH}$ binaries. We note that adopting a uniform rate of mergers might be an optimistic assumption for galaxies similar to the Milky Way. In fact, Galactic globular clusters appear to be exclusively old ( $\gtrsim 10$ Gyr) stellar systems (e.g., Rosenberg et al. 1999), implying that most $\mathrm{BH}$ mergers are likely to have occurred early on at high redshifts. However, this might not be the case in many external galaxies in which massive globular (like) clusters often appear to form in an ongoing and continuous process (Larsen \& Richtler 2000).

\subsection{Merger of binaries with stellar components}

We finally note that the results derived here could also apply to a variety of astrophysical systems. These include stellar binaries, starcompact object binaries (Mazeh \& Shaham 1979; Eggleton \& Kisseleva-Eggleton 2006) and binaries residing in galactic nuclei near massive BHs (Antonini et al. 2010a; Antonini \& Perets 2012; Prodan et al. 2013b). For these binaries GW radiation is likely not to play an important role. Their merger could be however induced by the Kozai mechanism combined with tidal friction.

These systems could also experience the non-secular dynamical evolution discussed here, which would largely increase the chance of collisions with respect to what was previously thought. Mergers of main sequence binaries are known to be a possible source of blue struggles in GCs (Perets \& Fabrycky 2009), they might produce a population of rejuvenated stars at the Galactic center (Antonini et al. 2011), and result in optical (e.g., Tylenda et al. 2011) and X-ray (Antonini et al. 2010b) transient events. The formation of type Ia supernovae could also be induced by similar processes (Katz \& Dong 2013). A more quantitative study of the evolution of moderately-hierarchical triples containing binaries with main-sequence star components and various types of stellar remnants will be the topic of future work.

We thank the referee for his/her valuable comments. We are grateful to C. Miller, H. Pfeiffer and S. Prodan for useful comments that helped to improve an earlier version of the manuscript. We acknowledge helpful discussions with Y. Lithwick and F. Will.

\section{REFERENCES}

Aarseth, S. 2012, MNRAS, 422, 841

Abadie, J., Abbott, B. P., Abbott, R., Abernathy, M., Accadia, T., Acernese, F., Adams, C., Adhikari, R., Ajith, P., Allen, B., $\&$ et al. 2010, Classical and Quantum Gravity, 27, 173001

Abramovic, A. et al. 1992, Science, 256, 325

Acernese, et al. 2008, Classical and Quantum Gravity, 25, 114045

Antognini, J. M., Shappee, B. J., Thompson T. A., \&

Amaro-Seoane, P. 2013, ArXiv

Antonini, F., \& Perets, H. 2012, ApJ, 757, 27

Antonini, F., Faber, J., Gualandris, A., \& Merritt, D. 2010a, ApJ, 713,90

Antonini, F. et al. 2010b, ApJ, 717, 795

Antonini F., Lombardi J., \& Merritt D., 2011, ApJ, 731, 128

Bailyn, C. D., Jain, R. K.; Coppi, P., \& Orosz, J. A. 1998, ApJ, 499, 367

Baker, J. G, Centrella, J., Choi, D. I., Koppitz, M. \& van Meter, J. 2006, Phys.Rev.Lett., 96, 111102

Banerjee, S.,Baumgardt, H., Kroupa, P. 2010, MNRAS, 402, 371 Belczynski, K., Kalogera, V. \& Bulik., T. A 2002, ApJ, 572, 407 Belczynski, K., Taam, R. E., Kalogera, V., Rasio, F. A., \& Bulik, T. 2007, ApJ, 662, 504

Binney, J. \& Tremaine, S. 1987, Galactic Dynamics (Princeton, NJ: Princeton University Press)

Blaes, O., Lee, M. H., \& Socrates, A. 2002, ApJ, 578, 775

Blanchet, L. 2006, Living Rev. Rel., 9, 4

Brassington, N. J. et al. 2010, ApJ, 725, 1805

Brodie, J. P., \& Strader, J. 2006, ARA\&A, 44, 193

Brown, D., \& Zimmenrman, P. J. 2010, Phys. Rev., 81, 4007

Campanelli, M., Lousto, C., Marronetti, P., \& Zlochower, Y. 2006, Phys. Rev. Lett., 96, 111101

Damour, T. \& Deruelle, N. 1985, Ann. Inst. Henri Poincaré Phys. Théor., Vol. 43, No. 1, p. $107-132,43,107$

Downing, J. M. B., Benacquista, M. J., Giersz, M., \& Spurzem, R. 2010, MNRAS, 407, 1946

Downing, J. M. B., Benacquista, M. J., Giersz, M., \& Spurzem, R. 2011, MNRAS, 416, 133

East, W. E., McWilliams, S. T., Levin, J., Pretorius, F. 2013, Physical Review, 87, 3004
Eggleton, P. P. \& Kisseleva-Eggleton, L. 2006, Ap\&SS, 304, 75 Fabrycky, D., \& Tremaine, S. 2007, ApJ, 669, 1298

Farr, W. M., Sravan, N, Cantrell, A., Kreidberg, L., Bailyn,

C. D. Mandel, I., \& Kalogera, V. 2011, ApJ, 741, 103F

Fehlberg, E. 1968, NASA Tech Rep, TR T-287

Ford, E. B., Kozinsky, B., \& Rasio, F. A. 2000, ApJ, 535, 385

Ford, E. B., Kozinsky, B., \& Rasio, F. A. 2000b, ApJ, 535, 385

Fryeg, C. L., Kalogera, Vassiliki 2001, ApJ, 554, 548

Hamers, A. S., Pols, O. R., Claeys, J. S. W., \& Nelemans, G. 2013, MNRAS, 430, 2262

Harry, G. M. \& LIGO Scientific Collaboration 2010, Classical and Quantum Gravity, 27, 084006

Heggie, D. C. 1975 , MNRAS, 173,729

Hellström, C., \& Mikkola, S. 2010, Cel. Mech. Dyn. Ast., 106, 143

Holman, M., Touma, J., \& Tremaine, S. 1997, Nature, 386, 254

Huerta, E. A., \& Brown, D. A. 2013, arXiv:1301.1895

Innanen, K. A., Zheng, J. Q.,Mikkola, S., \& Valtonen, M. J. 1997, AJ, 113, 1915

Kalogera, V., Belczynski, K., Kim, C., O'Shaughnessy, R., \& Willems B. 2007, Physics Reports, 442, 75

Katz, B., Dong, S., \& Malhorta, R. 2011, Phys. Rev. D, 107, 181101

Katz, B. \& Dong, S. arXiv:1211.4584

Kozai, Y. 1962, AJ, 67, 591

Kocsis, B., \& Levin, J. 2012, Physical Review, 85, 3005

Kulkarni, S. R., Hut, P. \& McMillan, S. 1993, Nature, 364, 421

Larsen, S. S., \& Richtler, T. 2000, A\&A, 354, 836

Lidov, M. L. 1962, Planet. Space Sci., 9, 719

Lithwick, Y. \& Naoz, S. 2011, ApJ, 742, 94

Maccarone, T. J., Kundu, A., Zepf, S. E., \& Rhode, K. L. 2007, Nature, 445, 183

Maccarone, T. J., Kundu, A., Zepf, S. E., \& Rhode, K. L. 2011, MNRAS, 410, 1655

Mardling, R. A., \& Aarseth, S. J. 2001, MNRAS, 321, 398

Mazeh, T. \& Shaham, J. 1979, A\&A, 77, 145

Merritt, D., Dynamics and Evolution of Galactic Nuclei, 2013, Princeton University Press 
Mikkola, S., \& Aarseth, S. J. 1993, Cel. Mech. Dyn. Astron., 57, 439

Mikkola, S., \& Merritt, D. 2006, MNRAS, 372, 219

Mikkola, S., \& Merritt, D. 2008, AJ, 135, 2398

Miller, M. C. \& Hamilton, D. P. 2002, ApJ, 576, 894

Miller, M. C. \& Hamilton, D. P. 2002, ApJ, MNRAS, 330, 232

Miller, M. C., \& Lauburg, V. M. 2009, ApJ, 692, 917

Morscher, M., Umbreit, S., Farr, W. M., \& Rasio, F. A. 2013, ApJ, 763, L15

Naoz, S., Farr, W. M., Lithwick, Y., Rasio, F. A., \& Teyssandier, J. 2011a, Nature, 473, 187

Naoz, S., Farr, W. M., Lithwick, Y., Rasio, F. A., \& Teyssandier, J. 2011b, arXiv1107.2414

Naoz, S., Bence K., Abraham Loeb, \& Nicolás Y. 2013, ApJ, 773 187

Seto, Naoki 2013, ArXiv

Narayan, R., Piran, T. \& Shemi, A. 1991, ApJ, 379, L17

O'Leary, R. M., Kocsis, B., \& Loeb, A. 2009, MNRAS, 395, 2127

O'Leary, R. M., Rasio, F. A., Fregeau, J. M., Ivanova, N., \&

O'Shaughnessy, R. 2006, ApJ, 637, 937

O'Shaughnessy, R., Kim, C., Kalogera, V., \& Belczynski, K. 2007, ApJ, 672, 479

Özel, F., Psaltis, D., Narayan, R., \& McClintock, J. 2010, ApJ, 725,1918

Perets, H. B. \& Fabrycky, D. C. 2009, ApJ, 697, 1048

Peters, P. C. 1964, Physical Review, 136, 1224
Pfeiffer, H. P. 2012, Class. Quant. Grav., 29, 124004

Portegies Zwart, S. F., \& McMillan, S. L. W. 2000, ApJ, 528, L17

Portegies Zwart, S. F. \& Yungelson, L. R., 1998, A\&A, 332, 173

Pretorius, F. 2005, Phys. Rev. Lett., 95, 121101

Prodan, S. \& Murray, N. 2012, ApJ, 747, 4

Prodan, S., Murray, N., Thompson, T. A. 2013, arXiv1305.2191

Prodan, S., Antonini, F., \& Hagai, Perets 2013 (in preparation)

Rosenberg, A., Saviane, I., Piotto, G., \& Aparicio, A. 1999 AJ, 118,2306

Sadowski, A., Belczynski, K., Bulik, T., Ivanova, N., Rasio, F. A.; O'Shaughnessy, R. 2008, ApJ, 676, 1162

Sigurdsson, S., Phinney, E. S. 1993, ApJ, 415, 631

Sigurdsson, S., Hernquist, L. 1993, Nature, 364, 423

Sippel, A. C. \& Hurley, J. R. 2013, MNRAS, 430, L30

Spitzer, L. 1987, Dynamical evolution of globular clusters (Princeton, NJ, Princeton University Press, 1987, 191 p.)

Strader, J., Chomiuk, L., Maccarone, T. J., Miller-Jones, J. C. A., \& Seth, A. C. 2012, Nature, 490, 71

Tylenda, R. et al. 2011, A\&A, 528, 114

Thompson, T. A. 2011, ApJ, 741, 82

Wen, L. 2003, ApJ, 598, 419

Woosley, S. E., Heger, A., \& Weaver, T. A. 2002, Reviews of Modern Physics, 74, 1015 